\title{
Shape and the Information in Medical Images: \\ A Decade of the Morphometric Synthesis
}

\author{
Fred L. Bookstein \\ University of Michigan, Ann Arbor, Michigan \\ fred@brainmap.med.umich.edu
}

\begin{abstract}
This keynote address reviews several techniques from morphometrics (the multivariate biometrics of shape) developed mainly in the context of medical image analysis over the last decade. The new techniques provide powerful tools for geometric tasks that arise in the course of most analyses of medical images in groups. These tasks include standardizing against Euclidean similarity transformations or shear transformations, encoding informative prior knowledge about shape variation, and detecting, testing, and visualizing linear statistical patterns of variation within or between groups. I review the features of the present toolkit, the standard underlying data models entailed, and some of the extensions that reach out to the additional information content of medical images for common clinical or scientific applications.
\end{abstract}

\section{Introduction}

Over the last decade there has been a remarkable convergence of several disciplines in one shared core of techniques, a morphometric synthesis, for the handling of biometric shape information as data.

The speed of this development caught most of us by surprise. The first announcement of the mathematical foundation (the Riemannian geometry of Kendall's shape space) came only in 1984 [1], via a long and difficult exposition making no reference at all to medical images. The initial publication of what is now taken to be the obvious associated multivariate statistical method [2] made no reference to this fundamental geometry except in one sentence of a contributed discussion. A crucial technique from interpolation theory, the thin-plate spline, was borrowed in 1987 [3] without reference to this or any other statistical context. (The same technique had already been applied by Terzopoulos and several colleagues to problems in image processing and computer graphics quite separate from this statistical arena.) In 1989 that multivariate statistics of shape was combined with the spline [4] for describing and decomposing group differences, still without acknowledgement of the geometric foundation. My full-length monograph [5] of 1991 continued to overlook the geometric roots of the relation between the spline and the statistics. As recently as five years ago, we still did not understand why the splines were so effective for the visualization of statistical effects on shape.

Only since then has the community of interested statisticians agreed on the source of this efficacy: the (elementary) theorem that the eigenfunctions of the spline are orthonormal in the Procrustes geometry of shape. This observation did not appear in the technical literature until $1995[6-7]$, and is only now being broadcast to readers not expert in mathematical statistics [8-10]. The most persuasive examples by which it is bought to the attention of these larger audiences, nevertheless, remain those drawn from the domain of medical image analysis: specifically, analyses of human brain shape variability in normal forms and in diagnosed disease states. Even though the canonical examples are thus medical image data sets, this keynote address is the first exposition of this synthesis for the professional community having the greatest use for it.

\section{The geometric foundation}

The core of the morphometric synthesis deals with landmark data, labelled points that correspond from instance to instance across all the images of a data set. Identification of landmarks is a strenuous and complex procedure requiring considerable prior anatomical understanding. The effort involved is justified afterward by the leverage that the information in their locations affords for subsequent scientific analyses. At present landmarks are almost always located manually; but work underway at Washington University and McGill suggests that it may soon be possible to locate them automatically. 


\subsection{Procrustes shape distance}

In ordinary language, the shape of an object is described by words or quantities that do not vary when the object is moved, rotated, enlarged, or reduced. The translations, rotations, and changes of scale we thereby ignore constitute the similarity group of transformations of the plane. When the "objects" are landmark sets, it turns out to be useful to say that their shape simply is the set of all point sets that "have the same shape." That is, we have formally defined the shape of a set of points as the equivalence class of that point set, within the collection of all point sets of the same cardinality, under the operation of the similarity group. (The more general linear groups $S L_{2}$ and $S L_{3}$ will concern us in Section 3.1.)

We need a distance measure for landmark shapes that have been defined in this way. Were it not for that complication of the similarity group, the obvious formula would involve the usual Pythagorean sum of squared distances between corresponding landmarks. It proves reasonable to define the squared distance between two shapes (that is, two equivalence classes) as the minimum of these sums of squares over representatives of the equivalence classes-over the operation of the group of similarities that shape is supposed to ignore. That is, the squared shape distance between one landmark set $\mathrm{A}$ and another landmark set $\mathrm{B}$ is the minimum summed squared Euclidean distances between the landmarks of $\mathrm{A}$ and the corresponding landmarks in point sets $\mathrm{C}$ as $\mathrm{C}$ ranges over the whole set of shapes equivalent to $\mathrm{B}$. For this definition to make sense, we have to fix the scale of $\mathrm{A}$. The mathematics of all this is most elegant (see [1]) just when the sum of squares of the points of $A$ around their center-of-gravity is constrained to be exactly 1 . A small adjustment of the definition, required for symmetry between $\mathrm{A}$ and $\mathrm{B}$, need not concern us here.

The steps in this computation follow down the rows of Figure 1. At the top are two quadrilaterals of landmarks presumed to arise from real images. Connect each landmark to the centroid of its own form and, for each form, rescale the sum of squares of the distances shown to unity (second row). Then (third row) translate one of the forms so that its centroid directly overlies the centroid of the other form. Finally, identify the rotation (third row right) that minimizes the sum of squares of the residual distances between matched landmarks; it can be computed analytically [5]. The squared Procrustes distance between the forms (fourth row) is the sum of squares of those residuals at its minimum. It is (approximately) the total area of the circles shown at the lower right, divided by $\pi$.
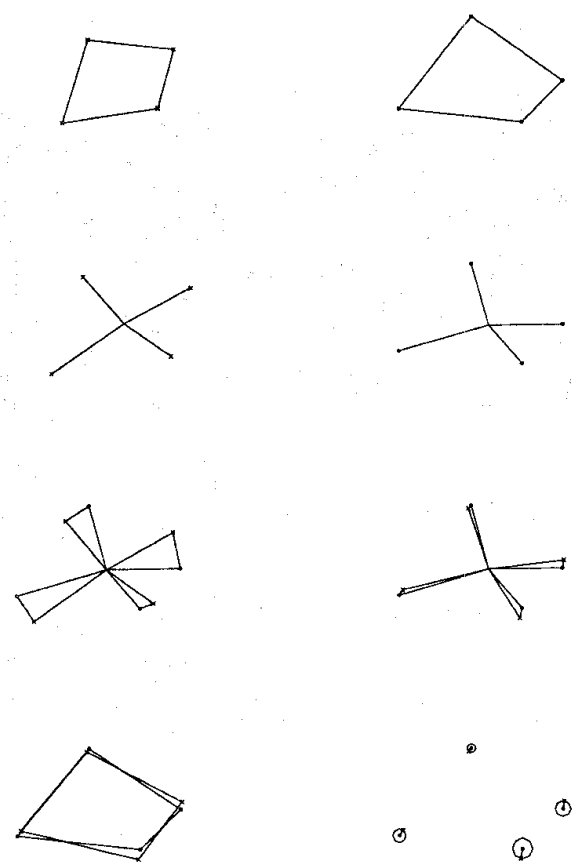

Figure 1. Procrustes shape distance for two quadrilaterals of landmarks (top row). Each form. is scaled separately (second row) to sum of squares 1 around its own centroid. After the centroids are superimposed (third row left), either form is rotated about the other (right) until the sum of squared distances between matched landmarks is minimized. (fourth row) The squared Procrustes distance between the original quadrilaterals is this minimum sum of squared distances between corresponding points; it is proportional to the sum of the areas of the circles drawn here.

Figure 2 typifies how landmark data arise from medical images. It is one image from a data set of 28 nearly midsagittal sections of human brains ( 14 schizophrenic and 14 not). The ten landmark points represent some reliable anatomical structures that intersect this plane.

\subsection{Averaging shapes}

The average of an ordinary list of numbers is their sum divided by their count. For averaging equivalence classes like these, we have recourse to a different characterization of that ordinary average: the least-squares fit to those numbers, the quantity about which they have the least sum of squared differences. From a distance between shapes, then, we inherit this notion of average shape directly. The necessary minimization is not too 
difficult [11]--for two-dimensional data, it comes from a closed-form eigenanalysis [12].

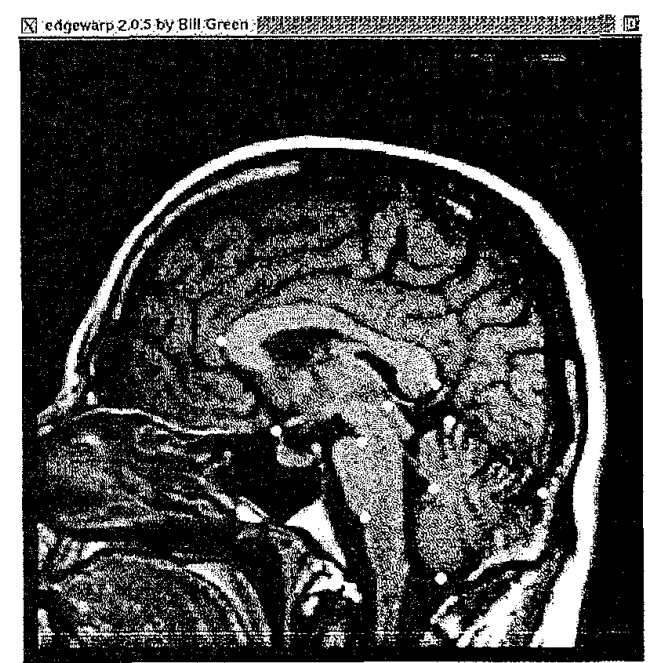

Figure 2. Landmark data ordinarily arise from the identification of particular named points in routine biomedical images. Here we identify ten landmarks in a parasagittal magnetic resonance image of a normal brain. Bottom to top: bottom of cerebellum, bottom of pons at medulla, tentorium at dura, obex of fourth ventricle, top of pons, optic chiasm, top of cerebellum, superior colliculus, splenium of corpus callosum, genu of corpus callosum.

For instance, for a data set of shapes like that of the dots in Figure 2, the average (Figure 3, left) looks just like a template for some landmark location scheme applicable to any case in particular. After we've computed the average, we can put each individual shape down over the average using the similarity transformation that made the sum-of-squares from the average a minimum for that particular case. There results the diagram at the right in Figure 3.

This tactic, far from being a mere graphical aid, is in fact the most crucial step in the geometric computations. The set of shapes construed as equivalence classes in this wise actually make up a Riemannian manifold, the Kendall shape manifold, with Procrustes distance as metric [1]. The manifold is of dimension $2 k-4: 2 k$ original Cartesian coordinates, decremented by the four degrees of freedom (two for translation, one for rotation, one for scale) of the similarity group of the plane. The tangent space to this manifold is likewise of dimension $2 k-4$ at every point. We will attend only to one of those tangent spaces, the one that is tangent at the sample average shape. The construction at the right in Figure 3 actually represents the projection of each shape of our sample onto the tangent space to that manifold at the sample average shape. Such a projection preserves the metric (in the small, in the vicinity of the sample average): for each pair of specimens, the summed squared distance between the representatives of the cases in Figure 3 is the same as their Procrustes shape distance. The $k$ coordinate pairs of that figure actually serve as a set of $2 k$ redundant coordinates for this $(2 k-4)$-dimensional tangent space.
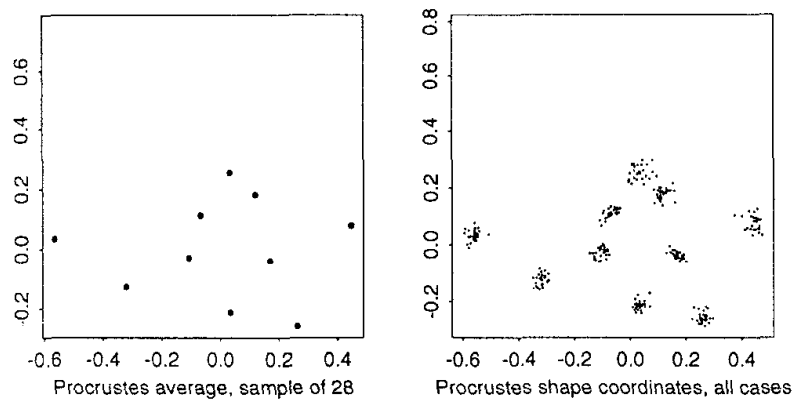

Figure 3. How Procrustes shape coordinates arise. (left) The Procrustes average of 28 shapes like that of the set of ten landmark points in Figure 2. (right) Procrustes shape coordinates come from fitting every individual case back over the sample average (left) by the procedure of Figure 1. These actually represent the orthogonal projection of the sample onto the tangent space to Kendall's shape manifold at the shape on the left (see text). The diagram is scaled to sumof-squares 1 around $(0,0)$; then both axes are in units of Procrustes distance per se.

\subsection{Multivariate statistical analysis}

An alternate way of construing tangent spaces provides the conceptual link to the next theme of the synthesis. The same tangent space to a Riemannian manifold that one initially intuits as a higher-dimensional plane touching a curving hypersurface, rather as a sphere rests on the ground, can be construed instead [13] as the space of all linear germs of scalar functions along curves through a point of the manifold. To the statistician, these functions are just what we mean by shape variables. That is, the tangent space construction of Figure 3 provides the setting for all possible linearized multivariate analyses of the information in the shapes of the landmark configurations. Furthermore, the usual multivariate sum-of-squares metric of that representation is the underlying Procrustes metric of 
the manifold, and so the multivariate statistics will be commensurate with the original Procrustes geometry. This series of steps is reviewed in more detail in [14].

For shapes that are concentrated in a small region of the full shape space (as any within-species sample of the shape of an organ is likely to be), one can carry out most of the ordinary maneuvers of multivariate statistical analysis-tests of group differences, correlations of shape with causes or effects, principal components analysis with respect to Procrustes distance-directly in terms of this basis for the tangent space. But because the basis is redundant, in this form it does not support techniques like regression that use matrix inversion; we will deal with this at Section 3.2 .

At the left in Figure 4, for example, is the computation of two averages for subsets of the 28 cases: one for fourteen patients actually diagnosed schizophrenic, the other for fourteen age- and sex-matched normals. This contrast is computed as a vector in the tangent space to Kendall's manifold. Again, it is drawn here by way of $k$ separate (but not orthogonal) projections onto $k$ planes within that space.

\subsection{Visualization via thin-plate spline}

There is a way to render that same vector of the tangent plane as one coherent graphical construction: a deformation grid. Imagine one of the averages, say, the normals (the dots), put down on ordinary square graph paper. Deform the paper so that the dots now fall directly over the other set of points, the triangles. The right panel in Figure 4 shows one plausible representation of what happens to the grid.

Naturally it matters what deformation one uses. The morphometric synthesis emphasizes one particular choice, the thin-plate spline, that minimizes yet another sum-of-squares. In this context, we are minimizing the summed squared second derivatives (integrated over the whole plane) of the map in the figure. Interpreted as the summed squared deviations of the shapes of the little squares from the shapes of their neighbors, it becomes a measure of local information in the mapping. That minimum actually turns out to be a quadratic form in the coordinates of the points of the target shape, with coefficients that depend on the starting shape $[4,8-10]$. This quadratic form, the bending energy, joins two others, the Procrustes metric and the empirical covariance matrix of the coordinates in Figure 3, to make up the geometrical substrate of the morphometric synthesis. Morphometrics seems unusual among branches of modern applied statistics in the centrality of this set of three quadratic forms rather than the usual two.
Please note that the spline is not claimed to be a realistic model for the correspondence of images between the landmarks. It is, rather, a visualization of directions in the tangent space to the shape manifold defined solely by the shapes of the landmark configurations. What is crucial about it, then, is that it is linear in the coordinates of the landmark shapes after projection, the coordinates of the right-hand panel in Figure 3. There are many other plausible parametric models for deformations of Euclidean space, but none of them seem to be linear in landmark coordinates in this same way. I will return to this concern of realism of deformations in Section 4.4.
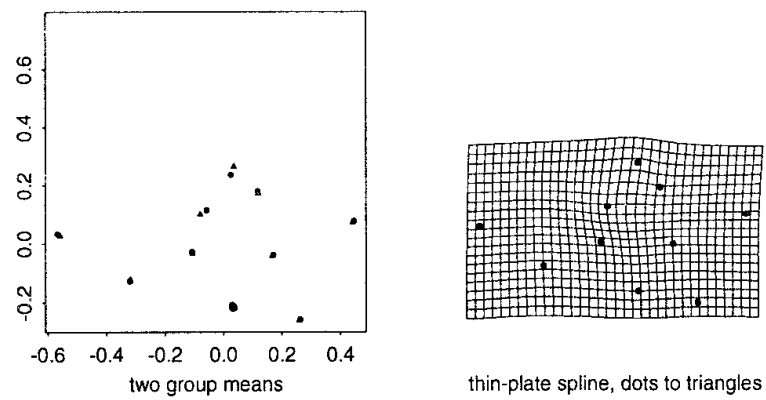

Figure 4. Statistics and visualizations in the tangent space to Kendall's manifold. (left) Example of statistical analysis in the tangent space: Average shapes for the two subgroups making up our original sample. Triangles, schizophrenics; dots, normals. The most striking discrepancy is at upper center. (right) The thin-plate spline is an interpolation function from one point set to another having the minimum variation (over the whole plane) of affine derivative. In the synthesis, it serves to visualize vectors in the tangent space at left. The grid here, the spline from the dots to the triangles at left, directly leads the eye to a local characterization of the shape difference under study. In other applications $[7-9]$ an equally obvious signal may appear at much larger scale.

\subsection{Testing a group difference}

The journals in which our typical clients publish are often overly concerned with statistical significance, improbability on a conventional "null hypothesis." For the simple two-group design here, there is a standard statistic owing to Goodall [15; see also 9] that often serves to reassure anxious reviewers and editors. Goodall's statistic, which applies equivalently to the two presentations paired in Figure 4, is based in the ratio between the squared Procrustes distance between group means (sum of squares of the distances between dots 
and triangles at the left in Figure 4) and the total of the squared Procrustes distances from each specimen to the grand mean (sum of the squares of the distances of the points from their landmark-specific centroids at the right in Figure 3). While the exact distribution of this ratio is a tabulated $F$ under one very symmetric set of assumptions, it is often more realistically taken as a permutation test [16]. For this computation, the observed value of the ratio I just mentioned is calibrated against the cumulative distribution of analogous ratios computed after group is assigned by (hundreds of) random permutation of the truth rather than according to the actual diagnosis case by case.

By either approach, the average shapes of schizophrenics and normals here differ at about the $2 \%$ level of statistical significance. (That is, we have a finding to report.) The consequences of such a verification for comparison of functional images will concern us presently.

\subsection{Three dimensions}

All this geometry goes over to three-dimensional data without any essential changes (except that things are much harder to draw). The characterization of Procrustes distance between shapes is the same (minimum sum-of-squares over the Euclidean similarity group when one form is constrained to sum-of-squares unity), and likewise the definition of the average shape. The dimension of the shape manifold, likewise the tangent space, is now $3 k-7$, and the Procrustes residuals (analogues to the points in Figure 3, right) now have 7 redundant coordinates, not 4 . The formalism of the spline goes over to three-dimensional data almost unchanged [5], and likewise all the multivariate statistical strategies.

\section{Shape variation between subjects}

The main import of this Morphometric Synthesis for medical image analysis is the praxis it supplies for coping with the non-invariance of the meaning of individual pixels - with shape variation between subjects. In this section I review the remaining statistical and graphical tools that apply to these problems.

The thin-plate spline applies to unwarp a data set of images to the average landmark configuration. Introduced in 1990, the splined unwarping uses the inverse of the thin-plate spline interpolant from sample mean landmark shape to each specimen of the sample. Image by image, pixel values are pulled back to the coordinate system of the mean landmark configuration, where they may be subjected to various further manipulations. Figure 5 shows the unwarping of the case of Figure 2 to the mean landmark configuration of the normal subgroup (dots in Figure 4). The averages by group of these unwarped images are presented in Figure 6.

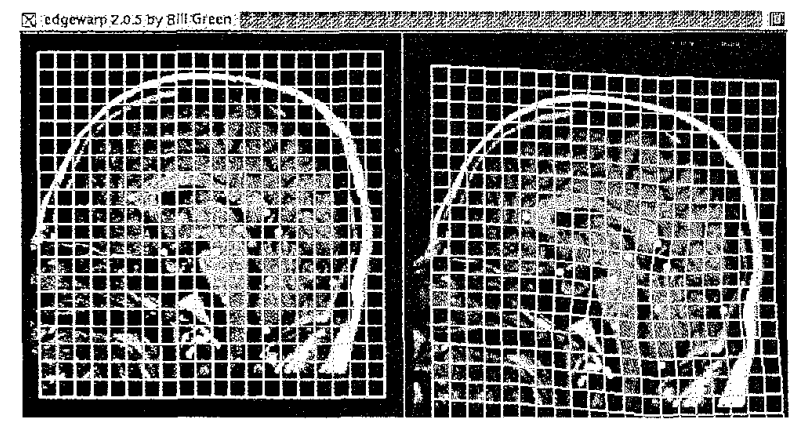

Figure 5. Unwarping of the image in Figure 2 to the normal average landmark configuration. Grid: thin-plate spline mapping, left $\rightarrow$ right. Unwarping: pixel pullback, right $\rightarrow$ left.

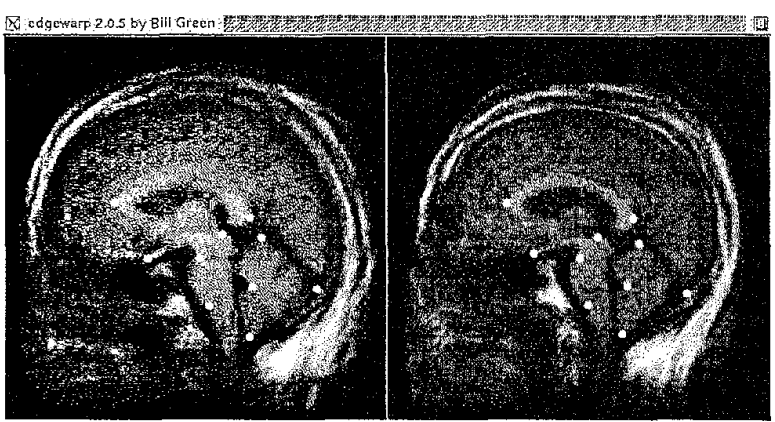

Figure 6. Average unwarped images, 14 per group. Each specimen is unwarped as in Figure 5 to its own average landmark configuration, and the resulting images summed pixelwise. Note the difference in shapes at the averages of splenium (right end of arch at center).

The import of the morphometric synthesis for medical image analysis follows mainly from the scientific power of these straightforward geometric maneuvers. The $2 k$-vector of landmark shape from Figure 3 behaves just like any other classic vector-valued biometric covariate, a list of associated values by which effects on the primary datum (in this case, the entire image) can be observed more precisely. But, in view of its extremely low dimension, the manipulation is more appropriately considered a relabeling of the pixels of the ground plane than any form of image processing per se. (For instance, the adjustment across Figure 5 does not alter the qualitative properties of any segmentation, but only the Cartesian coordinates at which the segments are located.) 
The context in which the morphometric synthesis is applicable to medical image analysis is thus not the consideration of images one at a time, as as for segmentation, or even two at a time, as in some more recent methods of deformable-template analysis, but their consideration in whole stacks of samples, for the extraction of patterns, typologies, forecasts, or causal understandings. The shape information, in other words, is intended from the outset to be combined with other information outside the image-group labels, clinical assessments, or coregistered images of another modality - toward the formulation or testing of quantitative biomedical hypotheses.

\subsection{Empirical components of shape variation}

One current community concern that the synthesis immediately resolves is the problem of uniform standardization. The same Procrustes geometry that optimally handles the four (in $3 \mathrm{~d}$, seven) nuisance parameters of the similarity group (translation, rotation, scale) extends directly to the additional two parameters of the general linear group $S L_{2}$ (in three dimensions, the additional five of $S L_{3}$ ). In the Procrustes space of $2 \mathrm{~d}$ data, the set of forms that derive from the sample average landmark configuration by uniform transformations (simple shears) lie on an ordinary (twodimensional) plane through the average shape. Every specimen has a uniform coordinate pair derived from Procrustes-orthogonal projection onto this subspace, and also a Procrustes-orthogonal residual from that projection, which is, rigorously, the geometrically unique nonuniform part of shape variation. The Pythagorean Theorem applies to this decomposition: squared Procrustes distance equals squared uniform distance plus squared nonuniform distance, and the two parts can be averaged and tested separately.

As the formulas for this term were not published until 1995, I will repeat them here. Let the Procrustes mean form (Figure 3 left), oriented with principal axes horizontal and vertical (as in the figures here), have coordinates $\left(x_{1}, y_{1}\right),\left(x_{2}, y_{2}\right), \ldots,\left(x_{k}, y_{k}\right)$, and let $\alpha=$ $\Sigma x_{i}^{2}$ and $\gamma=\Sigma y_{i}^{2}$ be the principal moments along those axes. Then the two elements of this two-vector may be taken as dot products of the Procrustes fit coordinates (Figure 3 , right), treated as a 20 -vector of real numbers, with the vectors $U n_{1}$ and $U n_{2}$ where

$$
\begin{gathered}
\lambda U n_{1}^{t}=\left(\left(\alpha y_{1}, \gamma x_{1}\right),\left(\alpha y_{2}, \gamma x_{2}\right), \ldots,\left(\alpha y_{k}, \gamma x_{k}\right)\right), \\
\lambda U n_{2}^{t}=\left(\left(-\gamma x_{1}, \alpha y_{1}\right),\left(-\gamma x_{2}, \alpha y_{2}\right), \ldots,\left(-\gamma x_{k}, \alpha y_{k}\right)\right) .
\end{gathered}
$$

Here $\lambda$ is the scaling factor $\sqrt{\alpha \gamma}$. The first of the vectors corresponds to Cartesian shears aligned with the $x$ axis, the second to Cartesian dilations along the $y$-axis.
Notice that these formulas are closed-the Procrustes uniform term is evaluated without further iteration.

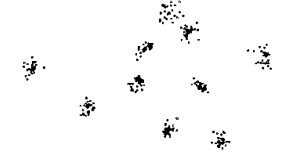

original Procrustes coordinates

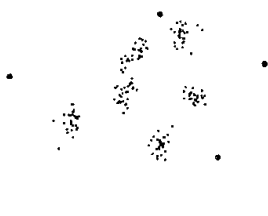

four-point registration

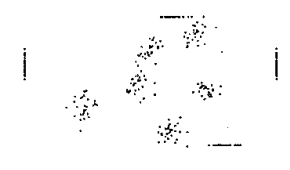

bounding-box-type coordinates

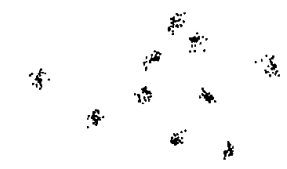

Procrustes affine-free coordinates
Figure 7. Counterintuitive behavior of plausible registration rules. (upper left) Original Procrustesfit coordinates as in Figure 3. (upper right) Adhoc attempt to remove the uniform part by alignment of the shape with a "bounding box." (lower left) "Refinement" of the bounding box, generated by nonlinear (inverse-spline) unwarping of the remaining landmarks when those on the bounding box are sent to their mean positions. Either of these innocuous-seeming protocols makes the scatter of registered points considerably worse than the original Procrustes registration that allowed no shape adjustments at all. (lower right) Optimal least-squares removal of the uniform term using the formulas in the text. Net Procrustes shape variance (summed moments of the little scatters) is necessarily reduced.

Standardization of the uniform part of shape variation by this least-squares procedure is guaranteed to optimally reduce the residual shape variance of any sample (Figure 7, upper left vs. lower right). Plausibleseeming approaches to uniform standardization not couched in this Procrustes geometry, such as the "bounding-box" routine simulated at upper right in the figure, are quite capable of adding unwanted shape variance, as they in fact do in this case. The paradox is not evaded, furthermore, by "nonlinear" treatment of the same four bounding landmarks (lower left: think of this as an analogue of Talairach-type registration on an arbitrary selection of scattered points). The error in all the ad-hoc techniques is a consequence of failure to apply least-squares to the information in the landmark configuration as a whole. Should the landmarks be known to have different precisions a priori, it is easy 
to adjust all these methods [5]. That heteroscedasticity is not particularly pressing here. Figure 8 shows the implications of such partial registrations for the averaged images that result. These and several other fallacies are wholly obscured when one works in units of raw centimeters in the original image space; they become obvious only when one evaluates methodological choices in the appropriate space of shape variation from the outset.

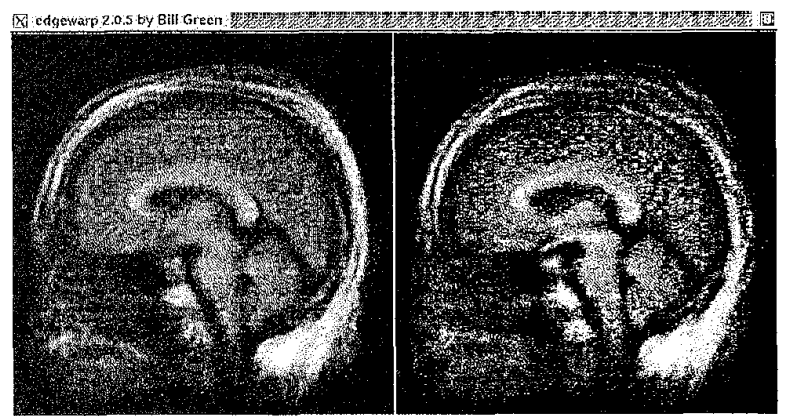

Figure 8. The difference between partial and full registrations is apparent in sharpness all over the averaged images except at shared landmark loci. Left, four-point registration (lower left, Figure 7); right, ten-point registration (average of the two frames in Figure 6 after a further unwarping to the average in Figure 3 ).

As these plausible partial registrations do not explain much of the shape variation in this actual sample, to what features should we turn? Because the Procrustes metric is retained alongside the sample covariance structure, the synthesis offers an optimal solution to this problem [7,10-14]: a set of empirical orthogonal components of bending that standardize images as efficiently as possible (in the Procrustes metric) for a given number of components. As Figure 9 shows, the first two of these components are not particularly aligned with the uniform part of the transformation. There is thus no good reason for any standardization [of this particular data set, anyway] to begin there, and no justification for any method of registration to end there. In this data set, while the first relative warp loads substantially on the uniform subspace, it is apparently more efficient to proceed with standardization of one particular small-scale bending term than with the second dimension of the uniform term. As it happens, that bending term is almost exactly aligned with the mean difference of the two samples: it is exactly the supervariance in which we were interested all along.

Principal components supply the optimal lower-dimensional linear reconstructions of empirical shape data vectors just as they do in any other application. In
Figure 10, we compare the ad-hoc four-point spline unwarping of Figure 7 to the optimal Procrustes registration using the same number of parameters.
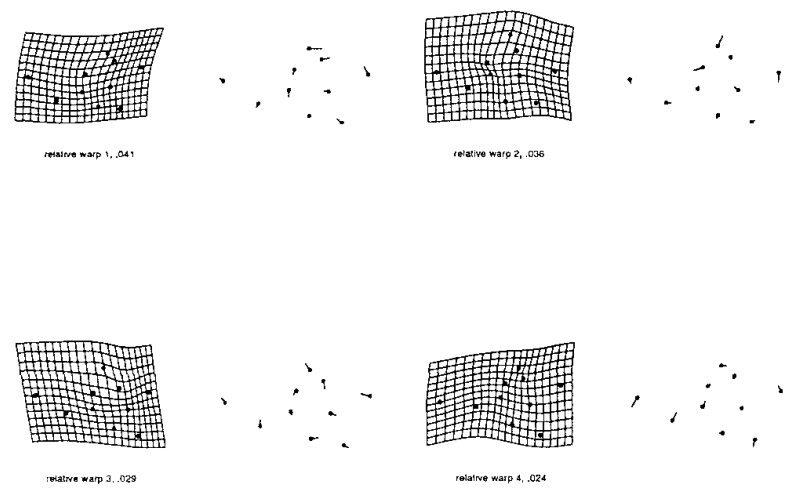

Figure 9. Empirical principal components (relative warps) of the full Procrustes shape scatter. Top row: first and second. Lower row: third and fourth. Each is shown both as a thin-plate spline and as a pattern of displacements in Procrustes space. Notice that the second component is very localized and strongly resembles the group difference in Figure 4.

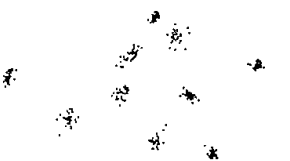

four-point fit, Procrustes

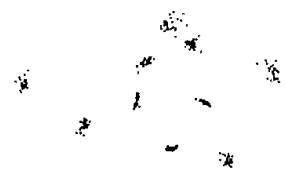

optimal four-df-adjusted Procrustes fit
Figure 10. Two four-df adjustments. (left) Procrustes reprojection of the ad-hoc four-point spline unwarping from Figure 7. (right) Optimal fourdimensional adjustment (projection): residual from the first four principal components of the full Procrustes scatter. Only $30 \%$ of the original 16dimensional variance remains.

\subsection{Geometric components a priori}

Some other approaches to deformable templates invoke a-priori bases for curving forms considered as functions. Sometimes these are copied directly out of textbooks (the Fourier decomposition for closed outlines, the Legendre polynomials for open curves, spherical harmonics for smooth convex blobs). Other bases occasionally suggested are functions of the mean or typical form: for instance, the normal modes of bending of the equivalent surface shells.

The morphometric synthesis includes one such basis, which, for our ten-point brain example, is shown 
in Figure 11: the eigenfunctions of the bending-energy quadratic form. These landmark displacements are actually orthonormal in the Procrustes metric underlying the geometry of the data. When projected onto both the $x$ - and $y$-axes of the Procrustes shape space, and supplemented by the uniform term just reviewed, they make up an orthogonal rotation of the original Procrustes residuals, Figure 3 (right), into a basis of the correct rank. Multivariate regressions and MANOVAs, for instance, go forward using this basis, and Procrustes distance is still preserved [14]. The bending energy of each of these normal modes serves as a useful index of its localization in the geometry of the original image plane. It is these features, not polynomials, that constitute the natural extension of the a-priori geometric basis beyond the obvious uniform term. For instance, for the ten-landmark data set here, the next two natural parameters are the $x$ - and $y$-projections of the surface at upper left in Figure 11, and the two after that, the projections of the surface to its right.
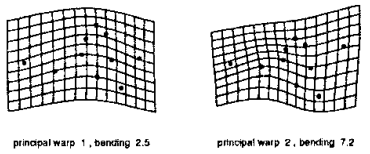
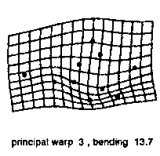

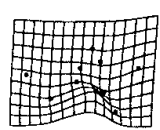

grincipal walp s. bending 21,

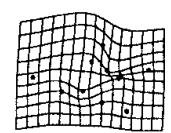

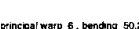

Figure 11. All seven principal warps of the sample average shape (Figure 3, left): eigenfunctions of the bending-energy quadratic form, drawn at Procrustes length 0.16 and interpreted as splined surfaces. Augmented by the uniform component, this basis provides a Procrustes-orthogonal rotation of the 16 nontrivial dimensions of the redundant basis at the right in Figure 3 .

Using this basis, we can at last understand why "bounding-box" adjustments like those in Figure 7 almost always make Procrustes scatters worse rather than better. By projecting the full sample of shapes onto the set of eight principal planes arising from the uniform term and these seven surfaces, we arrive at another ordination of which the uniform part is one single term (instead of being distributed over all the panels of the projection as in Figure 3 ). In this sample of brain shapes, the uniform term only accounts for only $20 \%$ of the total shape variance; the largest-scale nonuniform term (displacement of the center with respect to the anterior and posterior) actually accounts for more variation. The bounding box is a registration on an unnecessarily noisy estimate of this term; that noise propagates throughout the registered images, swamping any potential gain from the adjustment.

In some applications [7-8], the uniform or the first couple of nonuniform terms more or less exhaust the variation of shape space. Only in such cases are simplistic nonlinear registrations an efficient use of the algorithm's, or the investigator's, time. I have seen no evidence of any such a-priori low-dimensional statistical structure for brain data in either two or three dimensions. Whenever such structure is lacking, little can be gained by restriction of one's attention to any a-priori subspace of the shape tangent space. Still, the first four relative warps, Figure 9 , account for $70 \%$ of the total shape variation, considerably more than the ad-hoc procedure based on a "bounding box." Of course, this subspace is not known a priori.
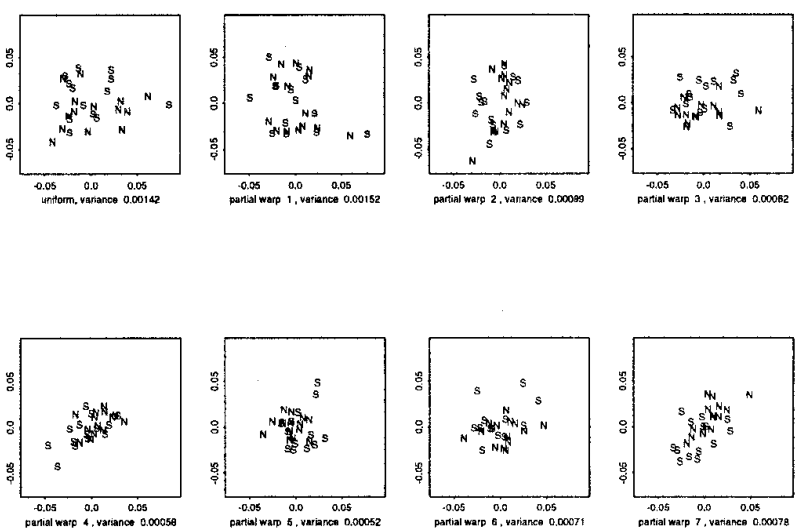

Figure 12. Decomposition of sample variation by partial warps (projections onto the orthonormal basis of Figure 11), together with the uniform component (upper, left). Of the total shape variance of .0072 , only about $20 \%$ is uniform, and less than $25 \%$ is aligned with the largest-scale nonlinear bending (Figure 11, upper left). $N$, normals; $S$, schizophrenics. All frames are commensurate in the Procrustes metric. The separation of $N$ 's and $S$ 's is largest in the panel at lower right, corresponding to the most focused dimension of warping. Axes are in the same units as Figure 3 .

Prior knowledge. Incorporating prior knowledge of shape variability is one of the main frontiers of medical image analysis. In the presence of sufficiently many 
cases, the representation by the full Procrustes landmark shape covariance structure is optimal for most multivariate purposes, such as linear discriminant function analysis. When samples are too small, or landmarks too numerous, to permit exploiting the full covariance matrix, any sublist of the first few empirical components or canonical variates provides an optimal basis of its order. The empirical orthogonal basis is also quite useful for describing the effects of any exogenous predictor of shape, such as group membership; it calls the scientist's attention to features that may suggest mechanisms, for example. In the example here, more than a third of the difference between schizophrenic and normal mean shapes is aligned with the last partial warp (Figure 11, lower right), but hardly any on the large-scale features that were adjusted out at the left in Figure 8; this "plausible" partial registration thus made the detection of group differences a good deal more difficult.

The "Procrustes sphere" against which all these distributions are calibrated actually corresponds to a statistical distribution of its own, the "null" of the Goodall $F[11]$. It is nearly spherical normal in the tangent. space, and corresponds to the origin of landmarks by a process of isotropic diffusion (Brownian motion) around their mean positions. In this version, the distribution is even applicable to a case of "all landmarks the same" diffusion of particles from the same origin, astronomical loci, etc. $[1,6]$.

\section{Future applications throughout medical image analysis}

The role of the landmark-driven methods in rationalizing registration rules and in generating descriptions of group shape variation and shape differences is wellunderstood by now. In this section I will sketch extensions, most of them topics of active current research, that probe a wider range of scientific and clinical applications of the same classes of images.

\section{1. "Statistical parametric images"}

In any structural data set there will be more information about structural differences than can be captured by landmarks. The remaining information can often be unearthed by further consideration of these landmarkdriven registrations as hints about further unwarping. The difference between images like those in Figure 6, for example, suggests that there is additional information along the lower boundary of corpus callosum, at the roof of the third ventricle. The bulb of the averaged schizophrenic splenium appears considerably thinner in an anteroposterior direction, as if it has been eroded from the front.

Such differences, because they pertain to alreadysharp boundaries of named structures, should be interpreted "horizontally"--as loci at which unwarping is evidently inadequate-rather than "vertically" as differences of mean gray level to be analyzed by $t$-ratio or another geometry-free statistic. Because the unwarping methods so very effectively concentrate the information from these very low-dimensional parameter spaces, the efficiency by which they extract additional geometric signals is far higher than anything available from analysis of pixel values "in place," which only deblurs rather than relabeling. In last year's Proceedings [17] I introduced a method for improving the spatial precision of unwarping by comparison of group mean images in this way, an example that will appear shortly [10] in a more expository context; I will not repeat the demonstration here.

A structure-driven unwarping can be applied as well to any other image that has been placed in register with the structural image: for instance, fMRI. In this context, the same language of "covariate correction" applies that I have already introduced. The landmarkdriven unwarping serves to substantially increase the precision of clinically or scientifically interesting comparisons arrived at with its aid. Increase in precision of unwarping can be translated into increased precision of regression slopes inferred or into reduction in sample size required for statistical significance of the patterms detected, whichever you, your reviewers, or the bureaucrats who fund you prefer. Unlike the usual biometric covariate adjustment, however, these covariates do not adjust the "dependent variable" (the functional image) linearly in its own (pixelwise) function space. The adjustment is, rather, a highly nonlinear manipulation in that space- a pixel relabeling-that has been made linear only by the powerful Procrustes machinery of the synthesis.

\section{2. "Endophrenology" (structure-function covariance)}

When structure is used as a covariate of function in this way, as with any other covariate adjustment, there may be group differences in the covariate (shape) and in the adjusted outcome (function) at the same time. In many studies, too, there will be very interesting correlations between structure and function. The permutation test for group difference introduced in Section 2.5 is a special case of a quite general approach to the testing of covariance structures relating landmark locations to any outside feature vectors, such as image 
contents or clinical aspects of a diagnosis. The pivotal statistic for the generalization is the first singular value of the matrix of covariances between the Procrustes shape coordinates and the vector of exogenous measurements. The same computation can be used to test the association of normalized image contents with putative causes or effects [18]. In that application, the amplitude of an image variation pattern substitutes for the Procrustes length of a shape change.

These two styles of analysis can be combined to analyze landmark shape, normalized image contents, and function in one ommibus least-squares procedure. Because this computation (which comes under the general heading of Partial Least Squares) is invariant against rotation of any block separately, findings in the shape space can be visualized equivalently as Procrustes shifts or as splines, whichever seems more congenial. Rotation of vectors of image contents (e.g., counts by regions-of-interest) or of exogenous vectors is ordinarily much more difficult to reify; typically, these are interpreted as "profiles" or "contrasts" instead.

\subsection{When structure is "missing"}

Perhaps the most important application of these crosscorrelation methods will be to the task of registering functional images. One often hears the argument that most functional images (e.g., PET images from cognitive studies of normals) are not accompanied by structural images, and hence must be unwarped using only parameters that are available from the functional image itself: in practice, only the very most reliable loci where source intensity drops to zero (cerebrospinal fluid, skull). But the crosscorrelation methods of Partial Least Squares [18] can be applied to a training set of landmarks from two images in adequate rigid registration - structure and function of the same cases - to produce a hierarchy of covariances of anatomical structure with the "landmarks" of the functional image. Regressions driven by those covariances, applied to new functional images, produce "expected" landmark locations that can, in turn, drive the usual unwarping to an average shape (using only the information in the functional image). In this way we may be able to simulate the power of single-subject $z$-pixel analyses for studies that actually pool normal subjects in large numbers. I am very eager to help someone try this approach soon.

\subsection{Information from curving form}

Landmarks are image features, but as a set they constitute a stringent abstraction of the information from that medical image. There is considerably more to be learned about shape and shape normalization from parts of boundaries between landmarks: curves in two dimensions, curves or surfaces in three. Elsewhere in this volume [19] I introduce a joint modification of the two main methods of the synthesis, Procrustes projections and the thin-plate spline, that seems to handle this extended class of data with striking effectiveness. The spline is used to relax claims of point-correspondence along the smooth manifolds upon which homologues are actually bound [20]; the Procrustes procedures continue to apply to describe interesting dimensions of the resulting feature spaces and to provide optimal detection of scientifically important differences.

I put forward these new models as a novel experimental link between multivariate statistics of actual sample differences, statistics that must be in low-dimensional spaces, and the enormously higher dimension of the warping representations produced by spatially distributed lattices. Models of that class appear to minimize mismatch energies (the Miller approach) or maximize correlation (the Evans approach) under a different geometry not apposite, as this one is, to linear statistical description of group differences or to structure-function correlations. In my view, the statistical interplay between vertical and horizontal analyses analysis of deformation vs. analysis of levels of graywill be the next great theme of the morphometric toolkit. It might be possible, for instance, to set a scale for accuracy of the spatially parameterized warps when they are viewed as residuals from globally specified spline schemes. At the next of these Workshops I look forward to being able to report far less tentatively on this new class of methods.

\section{Envoi}

The morphometric synthesis for landmarks and landmark-like data is now a nearly mature branch of applied statistics. These powerful new linearized procedures represent all the information content of an important abstraction from the image: the shapes of landmark configurations. The techniques, some optimal and some canonical, are all characterized or defended by theorems. Their importance corresponds to the growing importance of the aspect of medical image science with which they directly articulate: analyses of groups, types, or causes and effects-large data sets of images, over multiple subjects, considered all at once. The Human Brain Project, for instance, is particularly interested in this nexus, and supports Miller's work and Evans's alongside mine.

In this context of irreducible intersubject variation, the shape representations afforded by the synthesis, 
and the splines that visualize the linear space they span, are meant to be borrowed for medical informatics in the same respectful but routine way that one borrows the Cramer-Rao inequality, or the singular-value decomposition, or Student's $t$. These are constructs and formulas, not software modules and certainly not "black boxes." The basic tools I have reviewed-the Riemannian shape manifold, Procrustes tangent space, Goodall's $F$ and its permutation test, the spline visualization, the affine subspace, relative warps, Partial Least Squares-are optimal solutions, on hand in advance, for a host of informatic problems. There is usually no longer any gain to be had by speculating on these matters in the course of other studies. Occasionally one can do better in particularly problematic contexts, to be sure, but this basic toolkit is sturdy enough for most scientific applications and clinical typologies.

The techniques I have sketched here, accompanied by a greater variety of examples than I can yet supply, ought to become part of future textbooks in medical image analysis. I look forward, too, to equally canonical breakthroughs in the extended applications-statistical images, structure-function correlations, information from curving form--on which I speculated at the close.

Acknowledgement. Preparation of this contribution was supported by NIH grants DA-09009 and GM37251 to Fred L. Bookstein. The former grant is jointly supported by the National Institute on Drug Abuse, the National Institute of Mental Health, and the National Institute on Aging as part of the Human Brain Project. Images were analyzed by Bill Green's program package edgewarp, avallable by FTP from the directory pub/edgewarp on brainmap.med.umich. edu.

\section{References}

[1] D. G. Kendall. Shape-manifolds, procrustean metrics, and complex projective spaces. Bull. London Mathematical Society 16:81-121, 1984.

[2] F. L. Bookstein. Size and shape spaces for landmark data in two dimensions. Statistical Science 1:181-242, 1986.

[3] F. L. Bookstein. Toward a notion of feature extraction for plane mappings. Pp. 23-43 in C. de Graaf and M. Viergever, eds., Proceedings of the Tenth International Conference on Information Processing in Medical Imaging. New York: Plenum, 1988.

[4] F. L. Bookstein. Principal warps: Thin-plate splines and the decomposition of deformations. I.E.E.E. Trans. Pattern Analysis and Machine Intelligence 11:567-585, 1989.

[5] F. L. Bookstein. Morphometric Tools for Landmark Data. Cambridge University Press, 1991.
[6] K. V. Mardia. Shape advances and future perspectives. Pp. 57-75 in K. V. Mardia and C. A. Gill, eds., Proc. Current Issues in Statistical Shape Analysis, Leeds University Press, 1995.

[7] F. L. Bookstein. Metrics and symmetries of the morphometric synthesis. Pp. $139-153$ in K. V. Mardia and C. A. Gill, eds., Proc. Current Issues in Statistical Shape Analysis. Leeds University Press, 1995.

[8] F. L. Bookstein. The Morphometric Synthesis for landmarks and edge-elements in images. Terra Nova $7: 393-$ $407,1995$.

[9] F. L. Bookstein. Biometrics, biomathematics, and the morphometric synthesis. Bulletin of Mathematical Biology 58:313-365, 1995.

[10] F. L. Bookstein. Biometrics and brain maps: the promise of the Morphometric Synthesis. To appear in S. Koslow and M. Huerta, eds., Neuroinformatics: An Overview of the Human Brain Project. Progress in Neuroinformatics, vol. 1. Hillsdale, NJ: Lawrence Erlbaum, 1996.

[11] F. J. Rohlf and D. Slice. Extensions of the Procrustes method for the optimal superposition of landmarks. Systematic Zoology 39:40-59, 1990.

[12] J. T. Kent. Current issues for statistical inference in shape analysis. Pp. $167-175$ in K. V. Mardia and C. A. Gill, eds., Proc. Current Issues in Statistical Shape Analysis. Leeds University Press, 1995.

[13] J. Koenderink. Solid Shape. Cambridge, MA: M.I.T., 1990.

[14] F. L. Bookstein. Combining the tools of geometric morphometrics. Pp. 131-151 in L. Marcus et al., eds., Proceedings of the NATO Advanced Study Institute on Morphometrics. New York: Plenum, 1996.

[15] C. R. Goodall. Procrustes methods in the statistical analysis of shape. J. Royal Statistical Society B53:285-339, 1991.

[16] P. Good. Permutation Tests. New York: Springer-Verlag, 1994.

[17] F. L. Bookstein. How to produce a landmark point: the statistical geometry of incompletely registered images. Pp. 266-277 in R. Melter et al., eds., Vision Geometry IV.S. P. I. E. Proceedings, v. 2573, 1995.

[18] McIntosh, A. R., F. Bookstein, J. Haxby, and C. Grady. Multivariate analysis of functional brain images using Partial Least Squares. Neurolmage, in press.

[19] F. L. Bookstein. Landmark methods for forms without landmarks. This volume.

[20] W. D. K. Green. Spline-based deformable models. Pp. 290-301 in R. Melter, A. Wu, F. Bookstein, and W. D. K. Green, eds., Vision Geometry IV. S. P. I. E. Proceedings, vol. 2573,1995 . 